\title{
Demonstrating Quantum Coherence and Metrology that is Resilient to Transversal Noise
}

\author{
Chao Zhang $\odot,{ }^{1,2}$ Thomas R. Bromley, ${ }^{3, *}$ Yun-Feng Huang, ${ }^{1,2, \dagger}$ Huan Cao, ${ }^{1,2}$ Wei-Min Lv, ${ }^{1,2}$ Bi-Heng Liu, ${ }^{1,2}$ \\ Chuan-Feng Li, ${ }^{1,2, \hbar}$ Guang-Can Guo, ${ }^{1,2}$ Marco Cianciaruso, ${ }^{3,8}$ and Gerardo Adesso $\oplus^{3, \|}$ \\ ${ }^{1}$ Key Laboratory of Quantum Information, University of Science and Technology of China, \\ CAS, Hefei, 230026, People's Republic of China \\ ${ }^{2}$ CAS Center For Excellence in Quantum Information and Quantum Physics, University of Science and Technology of China, \\ Hefei, 230026, People's Republic of China \\ ${ }^{3}$ School of Mathematical Sciences and Centre for the Mathematics and Theoretical Physics of Quantum Non-Equilibrium Systems, \\ University of Nottingham, University Park Campus, Nottingham NG7 2RD, United Kingdom
}

(Received 24 July 2019; published 1 November 2019)

\begin{abstract}
Quantum systems can be exploited for disruptive technologies but in practice quantum features are fragile due to noisy environments. Quantum coherence, a fundamental such feature, is a basis-dependent property that is known to exhibit a resilience to certain types of Markovian noise. Yet, it is still unclear whether this resilience can be relevant in practical tasks. Here, we experimentally investigate the resilient effect of quantum coherence in a photonic Greenberger-Horne-Zeilinger state under Markovian bit-flip noise, and explore its applications in a noisy metrology scenario. In particular, using up to six-qubit probes, we demonstrate that the standard quantum limit can be outperformed under a transversal noise strength of approximately equal magnitude to the signal, providing experimental evidence of metrological advantage even in the presence of uncorrelated Markovian noise. This work highlights the important role of passive control in noisy quantum hardware, which can act as a low-overhead complement to more traditional approaches such as quantum error correction, thus impacting on the deployment of quantum technologies in real-world settings.
\end{abstract}

DOI: 10.1103/PhysRevLett.123.180504

Introduction.-Harnessing quantum effects holds the promise of revolutionizing information processing in ways that greatly surpass current approaches, including quantum computing, communication, and metrology [1]. However quantum resources are very fragile and practical realizations of quantum sensors and processors inevitably interact with their surroundings, eventually losing their nonclassical properties. In particular, the process of "decoherence" [2] stands as one of the major obstacles in realizing scalable quantum technologies. During the past two decades, numerous efforts have been invested to devise active noise control schemes [3-6]. Quantum error correction with feedback control $[3,4]$ provides the most promising scheme to combat arbitrary noise; however the excessive resource overhead keeps it beyond reach of current technology. A complementary approach is to develop passive noise control schemes, which are more affordable, by harnessing the natural resilience of quantum resources to specific noise. For example, placing a system in a decoherence-free subspace (DFS) can make it inherently immune to collective noises [7-9].

Quantum coherence, encapsulating the idea of superposition of quantum states, is a defining feature of quantum mechanics and also a crucial resource for quantum information processing [10]. Recently, the development of a rigorous resource framework for coherence [11-13] has brought it back to the limelight and motivated a number of studies [14-18]. Coherence is defined with respect to a particular reference basis, usually specified by the physics of the system under investigation [11]. As such, one may intuitively expect its resilience to depend on the direction along which the noise acts. Surprisingly, it has been observed that, under suitable conditions, the coherence in a multiqubit system (with respect to the computational basis) can remain exactly constant under independent bitflip noise acting on each qubit [18-21], in a process known as "freezing." This freezing phenomenon takes place despite the quantum state itself evolving due to the noise, highlighting a key difference to the DFS scenario.

It is intriguing to explore practical applications of frozen or more generally resilient coherence, particularly in quantum parameter estimation [22-24], for which coherence in the eigenbasis of the parameter-imprinting generator is an essential resource. It is well known that the precision of noise-free quantum metrology can beat the standard quantum limit (SQL) and achieve the Heisenberg limit (HL) by exploiting entangled probes, e.g., Greenberger-Horne-Zeilinger (GHZ) states. However, the quantum advantage is much more elusive in realistic environments in which the noise and the unitary evolution imprinting the parameter act on the probes simultaneously. In fact, there are a number of no-go results demonstrating 
that for most types of uncorrelated noise the asymptotic scaling is constrained to be SQL-like [25-29]. Nevertheless, it has been shown theoretically that, when the noise is concentrated along a direction perpendicular to the unitary dynamics (known as transversal noise), even if the noise is purely Markovian, a superclassical precision scaling in frequency estimation can be maintained by optimizing the interrogation time [30,31]. Note that for parallel Markovian noise, the uncorrelated and GHZ probes achieve exactly the same precision; thus no quantum advantage can be achieved [25].

In this Letter, we use a highly controllable photonic system as an experimental test bed to investigate the resilience of quantum coherence and metrology against transversal noise. We first demonstrate frozen quantum coherence in a 4-photon GHZ state prepared in both the computational and $\sigma_{x}$ bases and then subjected to Markovian bit-flip noise. We observe that the quantum Fisher information for estimating a phase encoded along the $\sigma_{z}$ basis is also frozen in the GHZ state prepared in the $\sigma_{x}$ basis. We then consider a frequency estimation task with additional bit-flip noise, which mimics a scenario of relevance for atomic magnetometry [32]. We demonstrate that the SQL can be surpassed using GHZ probes of up to 6 qubits, despite their exposure to noise of comparable strength to the signal.

Frozen quantum coherence and quantum Fisher information (QFI).-Coherence is marked by the presence of off-diagonal elements of a density matrix with respect to a particular basis. Incoherent states are classical mixtures with respect to the basis, corresponding to the set of diagonal density matrices. Given the set of incoherent states $\mathcal{I}$, the degree of coherence of a state $\rho$ can be quantified by how distinguishable $\rho$ is from $\mathcal{I}$, where a distance-based measure can be used to quantify distinguishability (see the Supplemental Material (SM. II A) [33] for further details).

It is important to study the dynamical evolution of coherence quantifiers given the inevitable interaction of quantum systems with their environments. References [18,19] identified dynamical conditions under which all distance-based coherence monotones can be frozen in a class of $N$-qubit states with maximally mixed marginals ( $M_{N}^{3}$ states). Time-invariant coherence has been demonstrated under these conditions in a NMR experiment [21]. Subsequently, it has been found that the relative entropy measure of coherence plays a special role in determining freezing conditions, since all coherence monotones are frozen if and only if the relative entropy is frozen [20]. Such a criterion can help us to identify other classes of initial states exhibiting frozen coherence.

GHZ states are widely used as resources for quantum information processing. This Letter investigates the dynamical conditions and applications of frozen coherence in $N$-qubit GHZ states, forming a complementary set to the canonical $M_{N}^{3}$ states for $N>2$. We first focus on a 4-qubit
GHZ state, prepared in either the computational $\sigma_{z}(0 / 1)$ basis $\left|G_{4}\right\rangle=(1 / \sqrt{2})\left(|0\rangle^{\otimes 4}+|1\rangle^{\otimes 4}\right)$ or $\sigma_{x}( \pm)$ basis $\left|G_{4}^{ \pm}\right\rangle=$ $(1 / \sqrt{2})\left(|+\rangle^{\otimes 4}+|-\rangle^{\otimes 4}\right)$, where $| \pm\rangle=(|0\rangle \pm|1\rangle) / \sqrt{2}$. We see in the following that these states can exhibit both frozen coherence and frozen QFI.

The GHZ states (preparation part in Fig. 1) are generated by combining two sandwichlike Einstein-Podolsky-Rosen (EPR) photon pairs [36] through a polarizing beam splitter (PBS) [37]. Both photon pairs are prepared in the state $(1 / \sqrt{2})(|H H\rangle+|V V\rangle)$, where $H(V)$ denotes the horizontal (vertical) polarization of photons and encodes the qubit values 0 (1). The fidelity of the prepared GHZ state is as high as $97.5 \%$ (see SM. IC [33] for further details). Hadamard gates, implemented as half-wave plates (HWPs) at $22.5^{\circ}$, can be used to transform the state $\left|G_{4}\right\rangle$ into $\left|G_{4}^{ \pm}\right\rangle$. Then, each photon is fed into a bit-flip noise channel (evolution part in Fig. 1, see SM. II B [33] for details).

At the output (detection part in Fig. 1), we perform full state tomography of the evolved states with different noise strengths, allowing a full analysis of the evolution. For each state, we calculate the $l_{1}$ norm of coherence and the relative entropy of coherence (SM. II A [33]) with respect to both $0 / 1$ basis and \pm basis, resulting in four quantities overall. Figure 2(a) illustrates each case, while Figs. 2(b) and 2(c) show the corresponding coherence dynamics.

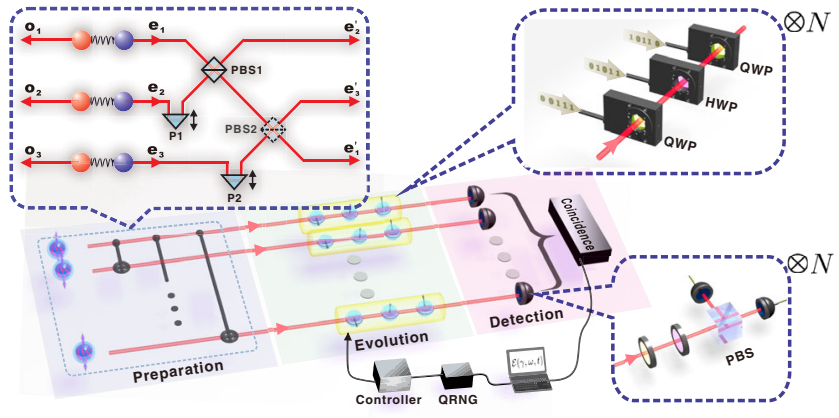

FIG. 1. The experimental setup consists of three main steps: preparation, evolution and detection. Ultraviolet laser pulses with a central wavelength of $390 \mathrm{~nm}$, pulse duration of $140 \mathrm{fs}$, and a repetition rate of $76 \mathrm{MHz}$ pass through three beamlike type-II spontaneous parametric down-conversion sources (not shown in the figure), and generate three EPR photon pairs in the state of $(|H H\rangle+|V V\rangle) / \sqrt{2}$. The three $e$ photons are fed into a multiphoton interferometer consisting of two PBSs. With postselection, a 6-photon GHZ state can be projected. By removing PBS2, the first two sources generate a 4-photon GHZ state, and the 3photon GHZ state can be generated by projecting one photon onto $(1 / \sqrt{2})(|H\rangle+|V\rangle)$ (see SM. I B [33] for details). After preparation, each photon passes through a channel which consists of three randomly rotated wave plates. All the wave plates are mounted on motorized rotation stages and controlled by a quantum random number generator (QRNG). By setting the parameters controlling the wave plates, various sources of noise can be simulated. In the measurement part, each photon is passed through a narrow-band filter and detected by a polarization analysis system. 
(a)

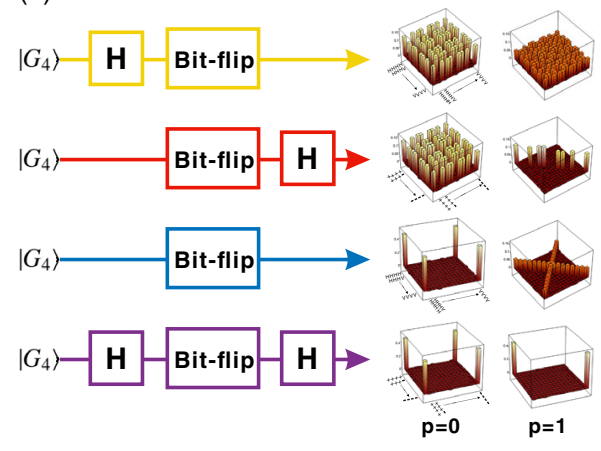

(b)

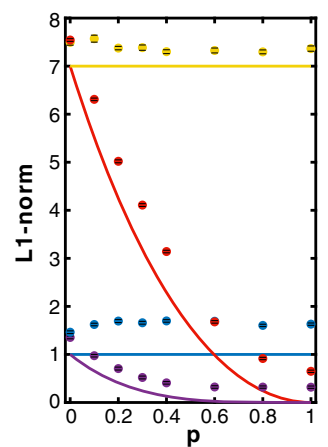

(c)

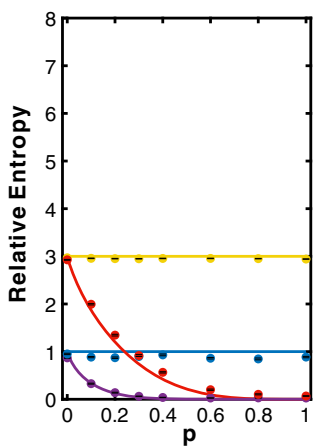

(d)

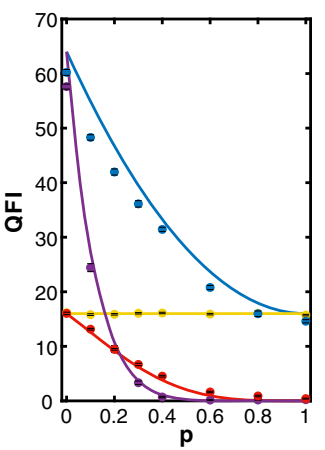

FIG. 2. (a) The schematic diagram of the four cases studied in our experiment: the yellow color (first line) for the case that the GHZ state is prepared in the \pm basis and coherence is calculated in the $0 / 1$ basis, denoted $\{ \pm, 0 / 1\}$; red (second line) for $\{0 / 1, \pm\}$; blue (third line) for $\{0 / 1,0 / 1\}$ and purple (fourth line) for $\{ \pm, \pm\}$. We use a Hadamard gate $(H)$ to represent the change between the $0 / 1$ and \pm bases. Shown on the right of the diagram are the real part of the reconstructed density matrices in the corresponding basis for $p=0$ and $p=1$, respectively. The right three panels show the measurement results (dots) of: (b) the $l_{1}$ norm of coherence, (c) the relative entropy of coherence, and (d) the QFI, along with theoretical predictions (solid lines) for the GHZ states during the evolution. The error bars are smaller than the marker size (the measured $l_{1}$-norm coherence is always larger than the theoretical value, due to the accumulation of the statistical errors for all off-diagonal elements of the density matrix).

When coherence is measured in the computational basis (yellow and blue lines), both coherence quantifiers remain constant for any value of noise strength $p$. On the other hand, the coherence measures in the \pm basis (red and purple lines) decay monotonically to zero. Note that the computational basis forms the eigenbasis of $\sigma_{z}$, which is orthogonal to the bit-flip noise generated by $\sigma_{x}$ [i.e., $\operatorname{Tr}\left(\sigma_{x} \sigma_{z}^{\dagger}\right)=0$ ]. This leads to the concept of freezing under transversal noise, which we now develop within the setting of metrology.

We first consider a simple model for noisy phase estimation with GHZ states, where bit-flip noise is assumed to act independently and before the parameter imprinting unitary. In this setting, the QFI, a figure of merit in quantum metrology, can be calculated directly through quantum state tomography using the experimental setup in Fig. 1. Suppose the unitary imprinting the unknown phase $\varphi$ to be $\exp \{-i \varphi H\}$ with a $N$-qubit Hamiltonian $H=\sum_{k=1}^{N} \sigma_{i}^{k}$, when $i=x(z)$, the unitary acts in a parallel (transversal) fashion to the bit-flip noise.

Figure 2(d) shows how the QFI depends on the noise strength for the noisy 4-qubit GHZ states of Fig. 1 when the noise is transversal (yellow and blue lines) and parallel (red and purple lines) relative to the parameter imprinting unitary. Interestingly, the noise dependence of the QFI is different from that of coherence. With transversal noise, the QFI is only constant for the GHZ state prepared in the \pm basis (yellow line); however it performs equally to the SQL corresponding to using an optimal uncorrelated probe $|++++\rangle$. Instead, for the GHZ state prepared in the $0 / 1$ basis, the QFI (blue line) does not remain constant and decays under noise, yet always exceeds the SQL. We see from this simple model that the best scenario for quantum metrology is to use a GHZ state prepared in a basis that coincides with the eigenbasis of the imprinting unitary, and moreover such that the noise is in a transversal direction to guarantee above-SQL performance. It is interesting to point out that coherence and QFI give a different ordering for such two initial states, showing that the coherence resource required for metrology relies on the means of encoding [16].

Noisy metrology.-We now consider a more realistic scenario in which the noise occurs simultaneously with the imprinting unitary. In this setting, we focus on estimation of a frequency $\omega$ imprinted as $\exp \{-i \omega t H\}$ with an $N$-qubit Hamiltonian $H=\frac{1}{2} \sum_{k=1}^{N} \sigma_{z}^{k}$. Following Refs. [30,31], we consider a dynamical evolution determined by a Lindbladtype master equation

$$
\frac{\partial \rho(t)}{\partial t}=\mathcal{H}(\rho)+\mathcal{L}(\rho),
$$

where $\mathcal{H}(\rho)=-i \omega[H, \rho]$ captures the unitary evolution and the Liouvillian $\mathcal{L}(\rho)$ describes the noise. We restrict to uncorrelated noise as this is the most likely form in experiments. Hence, $\mathcal{L}=\sum_{k} \mathcal{L}^{k}$ with single-qubit terms

$$
\mathcal{L}^{k}(\rho)=-\frac{\gamma}{2}\left(\rho-\alpha_{x} \sigma_{x}^{k} \rho \sigma_{x}^{k}-\alpha_{y} \sigma_{y}^{k} \rho \sigma_{y}^{k}-\alpha_{z} \sigma_{z}^{k} \rho \sigma_{z}^{k}\right),
$$

where $\gamma$ is the noise strength (identical for each qubit) and $\alpha_{i} \geq 0$ with $\alpha_{x}+\alpha_{y}+\alpha_{z}=1$. When $\alpha_{z}=1\left(\alpha_{x}=1\right)$, the noise is parallel (transversal) with respect to the unitary.

The precision in frequency estimation can depend on both the number of qubits $N$ of the probe and the interaction time $t$. In the noise-free setting (i.e., $\gamma=0$ ), both quantities can be increased to improve precision [38]. Increasing $t$ follows the intuitive notion that a longer interaction allows more information to be imparted upon the probe. However, the addition of noise causes the probe state to 
also deteriorate with time. This trade-off can result in an intermediate-time interaction being optimal for metrology.

We provide here an experimental verification that quantum probes can maintain superclassical performance in metrology despite the presence of noise, focusing on frequency estimation with transversal noise and interrogation-time optimization [30,31]. Our experimental setup remains as in Fig. 1. We use the $N$-qubit GHZ state $\left|G_{N}\right\rangle=$ $(1 / \sqrt{2})\left(|0\rangle^{\otimes N}+|1\rangle^{\otimes N}\right)$ in the computational basis as a probe and perform the metrological procedure for $N=1,2$, 3, 4, 6 qubits. Significant effort was made to prepare high fidelity probes: for the 2-, 4-, and 6-photon GHZ states, the fidelities were measured to be $0.9961 \pm 0.0002$, $0.9746 \pm 0.0027$, and $0.9059 \pm 0.0078$, respectively (see SM. I C [33] for more detail).

Transversal (bit-flip) noise corresponds to $\alpha_{x}=1$ in Eq. (2). To enact this noise experimentally, one can explicitly solve the master equation in Eq. (1) and obtain a single-qubit map expressed by a set of Kraus operators $\mathcal{E}_{\omega}(\rho)=\sum_{i=1}^{4} p_{i} K_{i} \rho K_{i}^{\dagger}$, where all $K_{i}$ are single-qubit unitary operations (see SM. III A [33] for detail). We can simulate the composite channel $\mathcal{E}_{\omega}^{\otimes N}$ by letting each photon pass through a HWP sandwiched by two QWPs. This combination can realize an arbitrary single-qubit unitary operation [39]. By randomly switching among the angle settings of the wave plates, we can realize each Kraus operator with desired probability (see SM. III B [33] for detail). Note that the random nature guarantees the Markov property of the simulated noise channel. In the experiment, we chose an identical noise and signal strength $\omega=\gamma=1$. Note that when the value $\gamma / \omega$ becomes larger, we require higher preparation fidelity of the GHZ probes to beat the SQL. To characterize the channel, we perform single-qubit process tomography with different evolution times (see SM. III B [33] for detail). The average process fidelity is measured to be $0.9952 \pm 0.0001$, confirming the reliable simulation of the channel.

The frequency $\omega$ is estimated based on the average of measuring a parity operator $P_{x}=\bigotimes_{k=1}^{N} \sigma_{x}^{k}$, which is optimal for GHZ probes in the noiseless case. The mean-squared error of $\omega$ can be deduced from error propagation, $\Delta^{2} \omega=\left[\left(\Delta^{2} P_{x}\right)_{\rho} /\left|\partial\left\langle P_{x}\right\rangle_{\rho} / \partial \omega\right|^{2}\right]$. Since $P_{x}^{2}=1$, it follows that $\Delta^{2} P_{x}=1-\left\langle P_{x}\right\rangle^{2}$. If the measurement is repeated $\nu$ times within a total time $T=\nu t$, the mean-squared error is reduced, as $\Delta^{2} \omega$ is inversely proportional to $\nu$. We consider the performance of frequency estimation with respect to the total time $T$, and can hence write

$$
\Delta^{2} \omega T=t \frac{1-\left\langle P_{x}\right\rangle_{t}^{2}}{\left|\partial\left\langle P_{x}\right\rangle_{t} / \partial \omega\right|^{2}}
$$

which is valid for the $T \gg t$ regime. As discussed above, there exists an optimized interrogation time $t^{\text {opt }}$ to minimize such a quantity, which we determine by theoretical calculation.

It is challenging to measure the partial derivative $\left.\left[\partial\left\langle P_{x}\right\rangle_{t} / \partial \omega\right]\right|_{\omega=1}$ experimentally. Here we use the fivepoint stencil method [40], to approximate the derivative (see SM. III C [33] for detail). For each $N$, we measured the average values of $P_{x}$ for five perturbations around $\omega=1$ with a spacing step of 0.1 or 0.2 ; the results are summarized in Fig. 3(a).

The mean-squared error of $\omega$ can then be calculated according to Eq. (3). Figure 3(b) shows the log-log plot of the mean-squared error as a function of the probe size $N$, from which we can see that a superclassical precision is demonstrated with GHZ probes of up to six qubits. Note that for parallel noise, there is no benefit in using a highly entangled GHZ state (see SM. III A [33] for detail). Figure 3(c) shows a plot of the Fisher information per photon/qubit [defined as $S_{N}=\left(N \Delta^{2} \omega T\right)^{-1}$ ] as a function of $N$, from which we can see for high quality GHZ probes $(N=1-4$, fidelity $>0.97)$ that the quantity increases with the entangled probe size. Instead, the Fisher information per qubit remains constant for unentangled probes. Note that the 2-photon Bell state performs equivalently to unentangled probes due to suboptimality of the parity measurement under bit-flip noise. For $N=6$, the results do not show that $S_{6}$ is larger than $S_{4}$ when taking into account uncertainties, mainly due to the relatively low
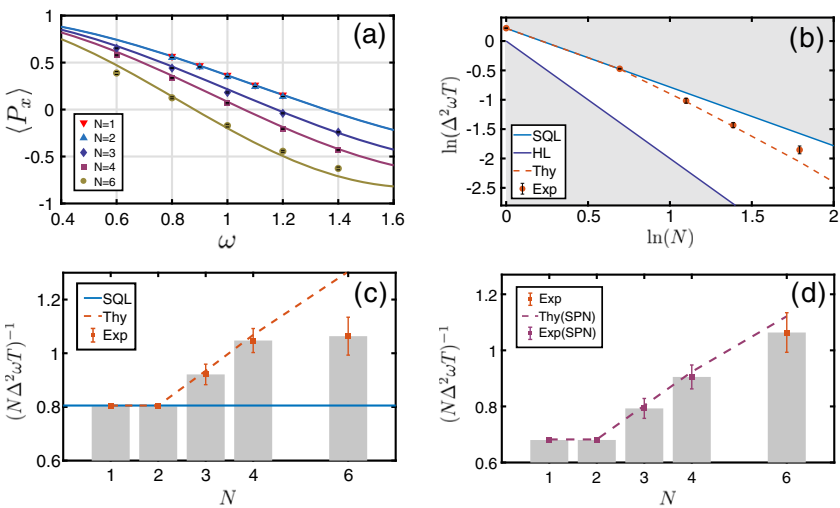

FIG. 3. (a) The measured average values of the parity operator $P_{x}$ for different probe size $N=\{1,2,3,4,6\}$. The optimal evolution times are $t^{\mathrm{opt}}=\{1.5490,0.7745,0.5684,0.4650$, $0.3508\}$, respectively. The solid lines show theoretical calculations using ideal probes and channels. Note that the red line $(N=1)$ is overlapped with the blue line $(N=2)$. The error bars are smaller than marker size. (b) The log-log plot of the meansquared error of $\omega$ determined by Eq. (3) as a function of the probe size. The results (red dots) are all sandwiched by the SQL and HL bounds, indicating an intermediate scaling. The red dashed line shows the theoretical predictions with ideal probes and channels. (c) Fisher information per photon as a function of $N$ (additional bars are drawn to aid illustration). (d) Fisher information per photon after adding $7 \%$ white noise in the $N=1-4 \mathrm{GHZ}$ probes. 
fidelity of the initial 6-photon GHZ state. To compare them with similar fidelity, we added further state preparation noise (SPN) in the $N=1-4 \mathrm{GHZ}$ probes. We consider white noise for simplicity, as it commutes with the incoherent channels. Our procedure was inspired by the effect of white noise on the observed statistics; we randomly flipped the measurement results of the parity operator with probability $p=\left(1-v_{\text {add }}\right) / 2$ to simulate adding further white noise $\rho_{\text {add }}=v_{\text {add }} \rho+(1-$ $\left.v_{\text {add }}\right)\left(\mathbb{I} / 2^{N}\right)$ [41]. According to the fidelity difference between $\left|G_{4}\right\rangle$ and $\left|G_{6}\right\rangle$, we set $v_{\text {add }}=0.93$ for $N=1-4$. After adding SPN, we observe the expected increasing with $N$ of the Fisher information per qubit [Fig. 3(d)]. In SM. III D [33], we give a numerical analysis showing that a fixed amount of noise in the initial probes does not impact how the metrological precision scales, while preparation noise that increases with $N$ can prevent superclassical scaling; if we treat such scaling preparation noise as a deviation from perfectly transversal noise in the channel, the result is similar to that discussed in Ref. [30]. In the SM. III E [33], we also determine the mean-squared error of $\omega$ by using the QFI of the evolved state $\mathcal{F}\left(\rho_{\omega}\right)(N=1-4)$, which also shows a superclassical precision scaling.

In conclusion, we have experimentally investigated the resilient effect of quantum coherence and QFI under transversal noise. In particular, we demonstrated that both quantities can be fully protected during the evolution in a 4photon GHZ state. We also investigated such resilience in a realistic metrology task where noise and parameter imprinting occur simultaneously. By configuring the signal Hamiltonian perpendicular to the noise, we demonstrated that our prepared GHZ probes can beat the SQL with up to 6-photon probes. For very pure probes (up to 4-photon), our results show that a quantum advantage in metrological scaling can survive even in the presence of uncorrelated Markovian noise, paving the way for scalable noisy metrology with only passive noise control. Future perspectives can be to combine our analysis with active error correction in metrology $[42,43]$. It would also be extremely attractive to find other applications which can harness the natural resilient effect of quantum resources against decoherence, especially in quantum computation.

This work was supported by the National Key Research and Development Program of China (Grant No. 2017YFA0304100), the National Natural Science Foundation of China (Grants No. 61327901, No. 11774335, No. 11734015, No. 11704371, and No. 11821404), Key Research Program of Frontier Sciences, CAS (Grant No. QYZDY-SSW-SLH003), the Fundamental Research Funds for the Central Universities (Grants No. WK2470000026 and No. WK2470000018), Anhui Initiative in Quantum Information Technologies (Grants No. AHY020100 and No. AHY070000), Science Foundation of the CAS (Grant No. ZDRW-XH2019-1), China Postdoctoral Science Foundation (Grant
No. 2017M612074), and the European Research Council (Starting Grant GQCOP No. 637352).

Note added.-Recently, we became aware of a related work [44], which demonstrates that local encoding provides a practical advantage for phase estimation in noisy environments.

*thomas.r.bromley@gmail.com

hyf@ustc.edu.cn

\$cfli@ustc.edu.cn

\$cianciaruso.marco@gmail.com

gerardo.adesso@nottingham.ac.uk

[1] L. Jaeger, The Second Quantum Revolution (Springer, New York, 2018).

[2] W. H. Zurek, Rev. Mod. Phys. 75, 715 (2003).

[3] P. W. Shor, Phys. Rev. A 52, R2493 (1995).

[4] D. Gottesman, arXiv:quant-ph/9705052.

[5] H. Rabitz, R. de Vivie-Riedle, M. Motzkus, and K. Kompa, Science 288, 824 (2000).

[6] L. Viola, E. Knill, and S. Lloyd, Phys. Rev. Lett. 82, 2417 (1999).

[7] D. A. Lidar and K. Birgitta Whaley, in Irreversible Quantum Dynamics, edited by F. Benatti and R. Floreanini, Lecture Notes in Physics Vol. 622 (Springer, Berlin, 2003), pp. 83-120.

[8] L.-M. Duan and G.-C. Guo, Phys. Rev. Lett. 79, 1953 (1997).

[9] P. G. Kwiat, A. J. Berglund, J. B. Altepeter, and A. G. White, Science 290, 498 (2000).

[10] A. Streltsov, G. Adesso, and M. B. Plenio, Rev. Mod. Phys. 89, 041003 (2017).

[11] T. Baumgratz, M. Cramer, and M. B. Plenio, Phys. Rev. Lett. 113, 140401 (2014).

[12] F. Levi and F. Mintert, New J. Phys. 16, 033007 (2014).

[13] A. Winter and D. Yang, Phys. Rev. Lett. 116, 120404 (2016).

[14] D. Girolami, Phys. Rev. Lett. 113, 170401 (2014).

[15] C. Napoli, T. R. Bromley, M. Cianciaruso, M. Piani, N. Johnston, and G. Adesso, Phys. Rev. Lett. 116, 150502 (2016).

[16] I. Marvian and R. W. Spekkens, Phys. Rev. A 94, 052324 (2016).

[17] C. Addis, G. Brebner, P. Haikka, and S. Maniscalco, Phys. Rev. A 89, 024101 (2014).

[18] T. R. Bromley, M. Cianciaruso, and G. Adesso, Phys. Rev. Lett. 114, 210401 (2015).

[19] M. Cianciaruso, T. R. Bromley, W. Roga, R. Lo Franco, and G. Adesso, Sci. Rep. 5, 10177 (2015).

[20] X.-D. Yu, D.-J. Zhang, C. L. Liu, and D. M. Tong, Phys. Rev. A 93, 060303(R) (2016).

[21] I. A. Silva, A. M. Souza, T. R. Bromley, M. Cianciaruso, R. Marx, R. S. Sarthour, I. S. Oliveira, R. Lo Franco, S. J. Glaser, E. R. deAzevedo, D. O. Soares-Pinto, and G. Adesso, Phys. Rev. Lett. 117, 160402 (2016).

[22] V. Giovannetti, S. Lloyd, and L. Maccone, Science 306, 1330 (2004). 
[23] V. Giovannetti, S. Lloyd, and L. Maccone, Phys. Rev. Lett. 96, 010401 (2006).

[24] V. Giovannetti, S. Lloyd, and L. Maccone, Nat. Photonics 5, 222 (2011).

[25] S. F. Huelga, C. Macchiavello, T. Pellizzari, A. K. Ekert, M. B. Plenio, and J. I. Cirac, Phys. Rev. Lett. 79, 3865 (1997).

[26] B. M. Escher, R. L. de Matos Filho, and L. Davidovich, Nat. Phys. 7, 406 (2011).

[27] R. Demkowicz-Dobrzański, J. Kołodyński, and M. Guţă, Nat. Commun. 3, 1063 (2012).

[28] J. Kołodyński and R. Demkowicz-Dobrzański, New J. Phys. 15, 073043 (2013).

[29] J. Kołodyński, Ph.D. thesis, University of Warsaw, 2014.

[30] R. Chaves, J. B. Brask, M. Markiewicz, J. Kołodyński, and A. Acín, Phys. Rev. Lett. 111, 120401 (2013).

[31] J. B. Brask, R. Chaves, and J. Kołodyński, Phys. Rev. X 5, 031010 (2015).

[32] W. Wasilewski, K. Jensen, H. Krauter, J. J. Renema, M. V. Balabas, and E. S. Polzik, Phys. Rev. Lett. 104, 133601 (2010).

[33] See Supplemental Material at http://link.aps.org/ supplemental/10.1103/PhysRevLett.123.180504 for technical details, including additional Refs. [34,35].
[34] S. L. Braunstein and C. M. Caves, Phys. Rev. Lett. 72, 3439 (1994).

[35] M. G. A. Paris, Int. J. Quantum. Inform. 07, 125 (2009).

[36] C. Zhang, Y.-F. Huang, Z. Wang, B.-H. Liu, C.-F. Li, and G.-C. Guo, Phys. Rev. Lett. 115, 260402 (2015).

[37] C. Zhang, Y.-F. Huang, C.-J. Zhang, J. Wang, B.-H. Liu, C.-F. Li, and G.-C. Guo, Opt. Express 24, 27059 (2016).

[38] B. L. Higgins, D. W. Berry, S. D. Bartlett, H. M. Wiseman, and G. J. Pryde, Nature (London) 450, 393 (2007).

[39] B. N. Simon, C. M. Chandrashekar, and S. Simon, Phys. Rev. A 85, 022323 (2012).

[40] https://en.wikipedia.org/wiki/Five-point_stencil.

[41] D. J. Saunders, A. J. Bennet, C. Branciard, and G. J. Pryde, Sci. Adv. 3, e1602743 (2017).

[42] E. M. Kessler, I. Lovchinsky, A. O. Sushkov, and M. D. Lukin, Phys. Rev. Lett. 112, 150802 (2014).

[43] S. Zhou, M. Zhang, J. Preskill, and L. Jiang, Nat. Commun. 9, 78 (2018).

[44] M. Proietti et al., preceding Letter, Phys. Rev. Lett. 123, 180503 (2019). 